\title{
S-duality in String Gas Cosmology
}

\author{
Savas Arapoglu ${ }^{1},{ }^{*}$ Ata $\mathrm{Karakci}^{2},{ }^{\dagger}$ and Ali Kaya ${ }^{1,2 \ddagger}$ \\ ${ }^{1}$ Feza Gürsey Institute, \\ Emek Mah. No:68, Cengelköy, Istanbul, Turkey \\ ${ }^{2}$ Bog̃aziçi University, Department of Physics, \\ 34342, Bebek, Istanbul, Turkey
}

(Dated: July 2, 2018)

\begin{abstract}
We consider a toy cosmological model in string theory involving the winding and momentum modes of $(m, n)$ strings, i.e. bound states of $m$ fundamental and $n$ D-strings. The model is invariant under S-duality provided that $m$ and $n$ are interchanged. The dilaton is naturally stabilized due to S-duality invariance, which offers a new mechanism of moduli fixing in string gas cosmology. Using a tachyon field rolling down to its ground state, we also point out a possible way of realizing a cosmological phase with decreasing Hubble radius and constant dilaton.
\end{abstract}

\section{INTRODUCTION}

String gas cosmology (SGC) [1] is a natural way of applying string theory to cosmology (for review see $[2,3]$ ). In addition to usual field theoretic excitations, in SGC one also considers new degrees of freedom of string theory and it turns out that the winding modes, which exist in the presence of compact directions, play an important cosmological role. The stringy T-duality invariance is the key symmetry which may resolve the initial big-bang singularity of standard cosmology. Moreover, the naive dependence of the tree level annihilation cross section of winding strings on the number of dimensions might explain the hierarchy between the observed three and internal directions [1] (however it seems there are some caveats in that argument [4-7] and a more complete understanding of string thermodynamics in an expanding universe is needed). In late times, SGC offers a natural mechanism to stabilize extra dimensions as a result of a balance between negative winding and positive momentum pressures [8]. Assuming that the cosmology is dominated by states which become massless at the special self-dual compactification radius, one can naturally avoid exceeding the phenomenological bounds on the energy density of the universe [9-11]. A string gas with fluxes is also capable of fixing the flux and the shape moduli [12].

SGC was developed in [13-15] to include higher dimensional $p$-branes that exist in string/M theory. Although, these are expected to annihilate in early times according to Brandenberger-Vafa mechanism [1], higher dimensional branes are needed to stabilize topologically nontoroidal cycles since winding string modes do not exist in the spectrum when the first homology class vanishes. Indeed, $p$-branes are capable of fixing not only the vol-

\footnotetext{
*arapoglu@gursey.gov.tr

† karakcia@boun.edu.tr

$\ddagger$ ali.kaya@boun.edu.tr
}

ume modulus of an internal Ricci flat manifold (radion) $[16,17]$ (see also [18-20]) but also the shape modulus of an internal torus they wrapped by the stress they generate [21].

Despite the fact that the late time stabilization of extra dimensions is natural in string/brane gas cosmology, dilaton stabilization (or stabilization of dilaton and radion simultaneously) is problematic [22, 23] (see however [24] and [23] for a solution by superpotentials). As Tduality plays a key role for the stabilization of extra dimensions, S-duality, which basically acts on dilaton, may assist in fixing dilaton. Indeed it was shown in [25] that compactifying the eleven dimensional supergravity on a circle the cosmological membrane production in the enhanced symmetry point can force the radius (and thus dilaton) towards the self S-dual point ${ }^{1}$.

Another motivation to consider S-duality in SGC comes from the recently proposed stringy mechanism to generate scale free cosmological perturbations seeded by thermal fluctuations in a strongly coupled Hagedorn phase [26-29]. According to [26-29], spacetime should be static in this meta-stable phase with infinite Hubble radius and it should be followed by the so called Hagedorn phase which has decreasing Hubble radius. During the Hagedorn phase cosmological perturbations exit the Hubble radius and freeze out. As criticized in [30], for this mechanism to really work out, the dilaton should be constant so that the string and Einstein frame Hubble parameters coincide. A possible way out of this criticism is to assume that the strongly coupled Hagedorn phase corresponds to a self S-dual state and thus dilaton should be equal to zero [29].

In the absence of a special symmetry that implies nonrenormalization (like supersymmetry), it seems difficult to give an effective field theory description of the strongly coupled Hagedorn phase since perturbative field equa-

\footnotetext{
${ }^{1}(m, n)$ strings that we consider in this paper also arise in the membrane compactification studied in [25].
} 
tions cannot be trusted. However, it should be possible to model the Hagedorn phase even if one enters into the strong coupling regime above the zero dilaton, since in that case one can apply an S-duality map to yield a weak coupling description.

Alternatively, one can also consider the possibility of having constant dilaton also in the Hagedorn phase. In that case, the equations of dilaton gravity reduce to that of usual Einstein's equations. Although it is not directly related to the main interest of this paper, namely S-duality, we also point out that using a tachyon field rolling down to its ground state it is possible to obtain a cosmological evolution with decreasing Hubble radius which can be matched to the static strongly coupled Hagedorn phase.

\section{S-DUALITY}

Let us start with the low energy effective action in 10dimensions

$$
S_{10}=\frac{1}{l_{s}^{8}} \int \sqrt{-g} e^{-2 \phi}\left[R+4(\nabla \phi)^{2}\right] .
$$

It is well known that (1) is invariant under S-duality transformation

$$
\begin{aligned}
& \phi \rightarrow-\phi \\
& g_{\mu \nu} \rightarrow e^{-\phi} g_{\mu \nu} .
\end{aligned}
$$

Since the string coupling constant $g_{s}$ is given by $g_{s}=e^{\phi}$, this map corresponds to a strong-weak coupling duality. Assuming a metric of the form,

$$
d s^{2}=-e^{2 A} d t^{2}+\sum_{i} e^{2 B_{i}} d \theta_{i}^{2}
$$

where the functions $A, B_{i}$ depend only on time $t$, the action (1) reduces to

$$
S_{10}=l_{s} \int d t e^{-\varphi-A}\left[\sum_{i} \dot{B}_{i}^{2}-\dot{\varphi}^{2}\right]
$$

where the shifted dilaton is defined as

$$
\varphi=2 \phi-\sum_{i} B_{i}
$$

Here we take the dimensionful coordinates $\theta_{i}$ to have length one in string units.

To add stringy matter to (1), the corresponding free energy $F$ should be coupled minimally to the string metric in the form of an effective Lagrangian [31]. Differing from [31], we assume that the free energy also depends on the string coupling constant, hence dilaton (or shifted dilaton), and the total action becomes

$$
S=S_{10}-\int d t e^{A} F\left(B_{i}, \varphi, \beta e^{A}\right) .
$$

The field equations following from this action in the proper time $A=0$ (and in string units $l_{s}=1$ ) read

$$
\begin{aligned}
& \dot{\varphi}^{2}-\sum_{i} \dot{B}_{i}^{2}=e^{\varphi} E, \\
& \ddot{B}_{i}-\dot{\varphi} \dot{B}_{i}=\frac{1}{2} e^{\varphi} P_{i}, \\
& 2 \ddot{\varphi}-\dot{\varphi}^{2}-\sum_{i} \dot{B}_{i}^{2}=-e^{\varphi} P_{\varphi},
\end{aligned}
$$

where

$$
E=F+\beta \frac{\partial F}{\partial \beta}, \quad P_{i}=-\frac{\partial F}{\partial B_{i}}, \quad P_{\varphi}=-\frac{\partial F}{\partial \varphi} .
$$

As a direct consequence of field equations, the coupled sources should obey the energy-momentum conservation equation

$$
\dot{E}+\sum_{i} \dot{B}_{i} P_{i}+\dot{\varphi} P_{\varphi}=0
$$

Here, we see that the shifted dilaton looks like an extra dimension, which is not surprising due to the 11dimensional origin of dilaton.

The conservation equation (9) implies that the entropy

$$
S \equiv \beta^{2} \frac{\partial F}{\partial \beta}
$$

is a constant of motion, hence the evolution is adiabatic. The temperature will adjust itself to yield constant entropy and one can write $\beta=\beta\left(B_{i}, \varphi\right)$. Therefore in the adiabatic approximation energy $E$ can be viewed as a function of $B_{i}$ and $\varphi$. In that case the pressures can be calculated $\mathrm{as}^{2}$

$$
P_{i}=-\frac{\partial E}{\partial B_{i}}, \quad P_{\varphi}=-\frac{\partial E}{\partial \varphi},
$$

which means that the total action becomes equivalent to

$$
S=S_{10}-\int d t e^{A} E\left(B_{i}, \varphi\right) .
$$

The action will be T-duality invariant provided

$$
E\left(B_{i}, \varphi\right)=E\left(-B_{i}, \varphi\right)
$$

On the other hand, from (2), S-duality invariance requires

$$
E\left(B_{i}, \phi\right)=e^{\phi / 2} E\left(B_{i}-\phi / 2,-\phi\right) .
$$

Note that in this equation we treat $E$ as a function of $\phi$, not the shifted dilaton $\varphi$.

\footnotetext{
2 To verify this note that when $\beta=\beta\left(B_{i}, \varphi\right)$ and $S=S_{0}, E=$ $\hat{F}+S_{0} / \beta$ where $\hat{F}\left(B_{i}, \varphi\right)=F\left(B_{i}, \varphi, \beta\left(B_{i}, \varphi\right)\right)$. Then $\frac{\partial E}{\partial B_{i}}=$ $\frac{\partial \hat{F}}{\partial B_{i}}-\frac{\partial \beta}{\partial B_{i}} \frac{S_{0}}{\beta^{2}}=\frac{\partial F}{\partial B_{i}}=-P_{i}$. The same manipulations for $\varphi$ gives (11).
} 


\section{STABILIZATION OF DILATON}

In type IIB string theory, an S-duality transformation (2) converts a fundamental string into a D-string. Actually, the action of S-duality in type IIB theory is more general than the mere strong-weak coupling map (2). It can be represented as an $S L(2, Z)$ transformation and by acting on the fundamental string one can obtain an $S L(2, Z)$ multiplet of strings characterized by two integers $m$ and $n$ [32]. An $(m, n)$ string can be viewed as the bound state of $m$ fundamental and $n$ D-strings [33]. The transformation (2) simply interchanges $m$ and $n$. In string frame the tension of the $(m, n)$ string is given by $[32]$

$$
T=T_{s} \sqrt{m^{2}+\frac{n^{2}}{g_{s}^{2}}}
$$

where $g_{s}$ is the string coupling constant and $T_{s}$ is the tension of the fundamental $(1,0)$ string determining the string length $l_{s}$. In seeking how S-duality acts in the context of string gas cosmology, it is clear that one should study a model involving $(m, n)$ strings.

The energy of an $(m, n)$ string winding a compact direction is proportional to its tension (15) times the radius of the circle. On the other hand, the energy of a momentum mode (either corresponding to a small vibration of a winding string or to an unwound string circling around that direction) is inversely proportional to the compactification radius. Note that the energy of the momentum mode should not depend on the tension (15). It should be measured in units of the corresponding angular coordinate parametrizing the circle and thus from (1) and (4) in terms of the string length $l_{s}$.

As a result, the string frame matter action for the winding and momentum modes of $(m, n)$ strings should take the form

$S=-\sum_{i} \int d t\left(E_{w} \sqrt{m^{2}+n^{2} e^{-2 \phi}} e^{A+B_{i}}+E_{m} e^{A-B_{i}}\right)$,

where the sum should go over compact directions, and $E_{w}$ and $E_{m}$ are constants characterizing winding and momentum energies, respectively. It is easy to see that the matter action is S-duality invariant with $m \leftrightarrow n$.

The S-duality transformation (2) is more manifest in Einstein frame defined by

$$
g_{\mu \nu}^{(E)}=e^{-\phi / 2} g_{\mu \nu}
$$

Writing the Einstein frame metric as (note that we are using the same letters for the metric components in Einstein frame, which should not be confused with (3))

$$
d s_{E}^{2}=-e^{2 A} d t^{2}+\sum_{i} e^{2 B_{i}} d \theta_{i}^{2}
$$

the total action becomes

$$
S_{E}=l_{s} \int d t e^{-A+\sum_{j} B_{j}}\left[\sum_{i} \dot{B}_{i}^{2}-\left(\sum_{j} \dot{B}_{j}\right)^{2}+\frac{1}{2} \dot{\phi}^{2}\right]
$$

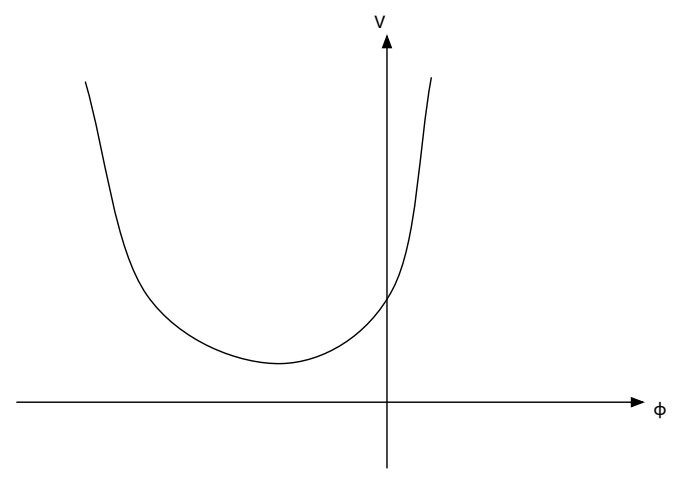

FIG. 1: The dilaton potential for $m>n$.

$$
-\sum_{i} \int d t\left(E_{w} \sqrt{m^{2} e^{\phi}+n^{2} e^{-\phi}} e^{A+B_{i}}+E_{m} e^{A-B_{i}}\right) .
$$

S-duality transformation in the Einstein frame is simply given by

$$
\phi \rightarrow-\phi
$$

and the invariance of the action $S_{E}$ is obvious provided $m \leftrightarrow n$. Note that S-duality leads to a potential ${ }^{3}$ for dilaton $V(\phi)=E_{w} \sqrt{m^{2} e^{\phi}+n^{2} e^{-\phi}}$ which has a global minimum (see figure).

In the proper time, the dilaton equation that follows from the above action reads (we set $l_{s}=1$ )

$$
\ddot{\phi}+\dot{k} \dot{\phi}=-\frac{1}{2} E_{w} \sum_{i} e^{B_{i}-k} \frac{m^{2} e^{\phi}-n^{2} e^{-\phi}}{\sqrt{m^{2} e^{\phi}+n^{2} e^{-\phi}}},
$$

where

$$
k=\sum_{i} B_{i}
$$

From (19) one sees that the constant dilaton

$$
\phi=\ln (n / m)
$$

is a solution. Moreover, (21) is also consistent with other field equations. Note that it is possible to stay in the weak coupling by choosing $m \gg n$.

To see that this is a stable point for dilaton and hence it is fixed, we first point out that $\dot{k}$ never vanishes, which follows from the variation of the action with respect to $A$. To preserve adiabatic approximation we impose $\dot{k}>0$ and thus $\dot{k} \dot{\phi}$ term in (19) acts like a frictional force that dumps the motion. Moreover, from the right hand side we see that when $\phi>\ln (n / m)$ there is a negative and when $\phi<\ln (n / m)$ there is a positive acceleration that

\footnotetext{
3 Actually $V(\phi)$ is not an honest potential since the action is not of the form $\int V \sqrt{-g}$. However, it still implies a non-trivial dilaton dependence of non-kinetic energy.
} 
force the equilibrium value. Indeed, looking for a small perturbation $\delta \phi$ around (21) we find that it obeys

$$
\ddot{\delta \phi}+\dot{k} \dot{\delta \phi}=-\left(E_{w} \sqrt{\frac{m n}{2}} \sum_{i} e^{B_{i}-k}\right) \delta \phi,
$$

which is similar to a dumped oscilator with time dependent friction and frequency.

To quantify these arguments and to see whether it is possible to stabilize both dilaton and radion simultanously let us consider $(1+d+p)$ dimensional split of the spacetime with the following metric

$$
d s_{E}^{2}=-d t^{2}+e^{2 B} d s_{d}^{2}+e^{2 C} d s_{p}^{2} .
$$

Here, $d s_{d}^{2}$ and $d s_{p}^{2}$ are flat metrics on $R^{d}$ and $T^{p}$, playing the roles of the observed and internal dimensions. From the Einstein frame action it is possible to obtain the following field equations

$$
\begin{aligned}
\ddot{\phi}+\dot{k} \dot{\phi} & =-\frac{p}{2} E_{w} e^{C-k} \frac{m^{2} e^{\phi}-n^{2} e^{-\phi}}{\sqrt{m^{2} e^{\phi}+n^{2} e^{-\phi}}}, \\
\ddot{C}+\dot{C} \dot{k} & =F_{m}-\frac{(d-5)}{4} F_{w}, \\
\ddot{B}+\dot{B} \dot{k} & =\frac{p}{4} F_{w}
\end{aligned}
$$

together with the initial constraint

$$
\dot{k}^{2}=d \dot{B}^{2}+p \dot{C}^{2}+\frac{1}{2} \dot{\phi}^{2}+2 p F_{w}+2 p F_{m},
$$

where the functions $F_{w}$ and $F_{m}$ are given by

$$
\begin{aligned}
& F_{w}=\frac{E_{w}}{2} \sqrt{m^{2} e^{\phi}+n^{2} e^{-\phi}} e^{-k+C}, \\
& F_{m}=\frac{E_{m}}{2} e^{-k-C} .
\end{aligned}
$$

Note that $k=d B+p C$ and $d+p=9$.

For $d>5$, there is a special solution with constant dilaton and radion given by

$$
\phi=\ln (n / m), \quad B=\frac{2}{d} \ln (\alpha t), \quad C=C_{0},
$$

where

$$
\begin{aligned}
& e^{2 C_{0}}=\frac{8 E_{m}}{(d-1) E_{w} \sqrt{2 m n}}, \\
& \alpha^{2}=\frac{d p E_{w} \sqrt{2 m n}}{16} e^{(1-p) C_{0}} .
\end{aligned}
$$

To verify stabilization let us consider small perturbations around this background which obey

$$
\begin{aligned}
& \ddot{\delta \phi}+\frac{2}{t} \dot{\delta \phi}+\frac{8}{d} \frac{\delta \phi}{t^{2}}=0, \\
& \ddot{\delta C}+\frac{2}{t} \dot{\delta} \dot{C}+\frac{4(d-5)}{d p} \frac{\delta C}{t^{2}}=0, \\
& \ddot{\delta B}+\frac{4}{t} \dot{\delta B}+\frac{2}{t^{2}} \delta B+\frac{2 p}{d} \frac{\delta C}{t}+\frac{(2 p-2)}{d} \frac{\delta C}{t^{2}}=0 .
\end{aligned}
$$

As a final comment we would like to point out a possible way of getting a decreasing Hubble radius in string theory. This should mimic the cosmological evolution in the Hagedorn phase [29], following the static strongly coupled Hagedorn phase. We assume that the dilaton

We see that $\delta \phi$ and $\delta C$ are nicely decoupled. The corresponding linearly independent solutions for them can be found as

$$
\frac{\cos \left[\ln \left(\frac{t \sqrt{4 \lambda-1}}{2}\right)\right]}{\sqrt{t}}, \frac{\sin \left[\ln \left(\frac{t \sqrt{4 \lambda-1}}{2}\right)\right]}{\sqrt{t}},
$$

where $\lambda=8 / d$ for $\delta \phi$ and $\lambda=4(d-5) /(d p)$ for $\delta C$, and in each case $\lambda>1 / 4$. Thus both $\delta \phi$ and $\delta C$ fall for large $t$ as $1 / \sqrt{t}$, which proves that they are stabilized. Similarly, it can be seen from (29) that the perturbation $\delta B$ also goes like $1 / \sqrt{t}$ as $t \rightarrow \infty$.

One may think that a cosmology dominated by $(m, n)$ strings of one type is not realistic. Indeed, $(m, n)$ strings have a larger tension compared to, say, $(1,0)$ or $(0,1)$ strings, which become lightest strings in Einstein frame at weak and strong couplings, respectively. However, assuming that the numbers of fundamental and D-strings do not change and, for the moment, they are equal to each other, then it is energetically more favorable to form $(1,1)$ strings, since the tension of a $(1,1)$ string is less than the sum of the tensions of $(1,0)$ and $(0,1)$ strings. Similarly, when the ratio of the numbers is $m / n$, the cosmology will be dominated by $(m, n)$ strings.

The restriction $d>5$ on the number of observed dimensions in the above toy model can easily be circumvented by including higher dimensional S-duality related branes. For instance, in type IIB string theory one can consider a bound state of $m$ Dirichlet and $n$ solitonic fivebranes which has the following tension in the Einstein frame [33]

$$
T=T_{5} \sqrt{m^{2} g_{s}+\frac{n^{2}}{g_{s}}} .
$$

As for strings, the duality map (18) interchanges $m$ and $n$. One can consider $(1+3+5+1)$ dimensional split of the spacetime where $(m, n)$ fivebranes and strings are wrapping over the five and the one dimensional subspaces of the six dimensional toroidal internal space, respectively. It can be verified that in this S-duality invariant model both radion and dilaton are fixed in a three dimensional observed space, where again $\phi=\ln (n / m)$.

The above results indicate that S-duality can play a crucial role in SGC, especially in the late time stabilization of dilaton. For early time applications, it seems a detailed information about the free energy of the string gas, particularly its dependence on the string coupling constant, is needed.

\section{DISCUSSION}


and internal dimensions are already stabilized leaving a four dimensional Einstein theory. A decreasing Hubble radius necessarily implies accelerated expansion, but the opposite is not correct. Therefore, it seems harder to obtain former compared to the acceleration. Indeed, if the spatial sections of the universe are flat, one can only get a decreasing Hubble radius by violating the null energy condition, i.e. $\rho+P<0$.

One method of obtaining acceleration in string theory is via a tachyon field $T$ rolling down to its ground state [34]. The tachyon potential $V(T)$ has a positive maximum $V_{0}$ at $T=0$ and a minimum at $T=T_{0}$ with $V\left(T_{0}\right)=0$. The Lagrangian is given by

$$
\mathcal{L}=-V(T) \sqrt{1-\dot{T}^{2}} .
$$

Considering the evolution of tachyon field from $T=0$ (with a small initial velocity) towards the minimum at $T=T_{0}$, one sees that $\dot{T}$ increases in time. From the corresponding energy momentum tensor it follows that for $\dot{T}^{2}<2 / 3, \rho+3 P<0$ which implies acceleration [34]. However, in this interval $\rho+P>0$ which gives an increasing Hubble radius. Thus the tachyon field alone cannot yield a decreasing Hubble radius.

Actually, there is another problem here which is also crucial for our discussion. From field equations one can see that during this accelerating phase the expansion speed can never vanish. Therefore, it is not possible to match this evolution to the static strongly coupled Hagedorn phase. To cure this difficulty one should essentially try to slow down the expansion speed. To that effect, the easiest madification is to assume that the space is positively curved rather than being flat. Taking the spacetime metric as

$$
d s^{2}=-e^{2 A} d t^{2}+e^{2 B} d \Omega_{3}^{2},
$$

where $d \Omega_{3}^{2}$ is the unit round metric on the three sphere, and coupling the tachyon field with the canonical potential $V(T)$, one obtains the action

$$
\begin{aligned}
S_{4} & =l_{p} \int d t e^{-A+3 B}\left(-6 \dot{B}^{2}\right)+6 \sigma e^{A+B} \\
& -\int d t e^{A+3 B} V(T) \sqrt{1-e^{-2 A \dot{T}^{2}}},
\end{aligned}
$$

where $\sigma=1$ and that term is related to the curvature of the sphere. Varying with respect to $A, B$ and $T$ one finds (we set $l_{p}=1$ )

$$
\begin{aligned}
& \dot{B}^{2}=-\sigma e^{-2 B}+\frac{V}{6 \sqrt{1-\dot{T}^{2}}}, \\
& \ddot{B}=\sigma e^{-2 B}-\frac{V \dot{T}^{2}}{4 \sqrt{1-\dot{T}^{2}}}, \\
& \frac{d}{d t}\left(\frac{V}{\sqrt{1-\dot{T}^{2}}}\right)=-3 \dot{B} \frac{V \dot{T}}{\sqrt{1-\dot{T}^{2}}} .
\end{aligned}
$$

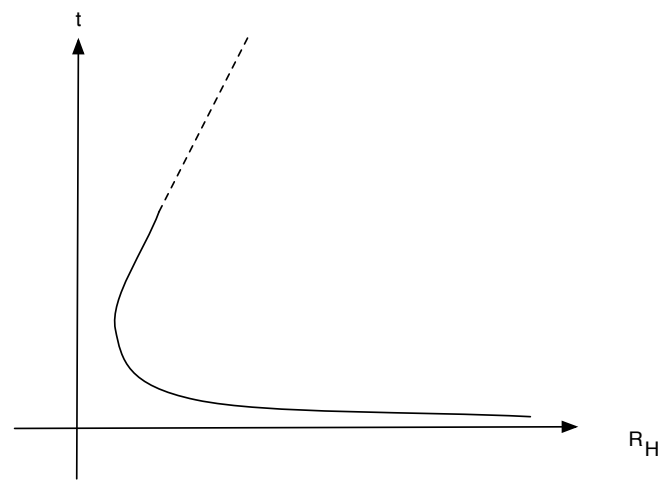

FIG. 2: The time evolution of the Hubble radius in the tachyon cosmology with a spherical space.

Note that, although the curvature of the sphere ( $\sigma$-term) contributes negatively to the expansion speed $\dot{B}$, it increases $\ddot{B}$, which is precisely what we were looking for.

Since we would like to match the background to the static strongly coupled Hagedorn phase, we assume that initially at $t=0, \dot{B}(0)=0$. For the tachyon field we impose $T(0)=0$ and take a small positive initial velocity, i.e $0<\dot{T}(0) \ll 1$ (assuming $\dot{T}(0)=0$ gives the Einstein static universe which is unstable). Then from (35) and (36) one sees that $\ddot{B}(0)>0$. Recalling that the Hubble radius $R_{H}$ is given by $R_{H} \sim 1 / \dot{B}$, it decreases initially.

To understand the succeeding evolution we note that since $\ddot{B}+\dot{B}^{2} \sim\left(1-3 \dot{T}^{2} / 2\right)$ the acceleration stops when $\dot{T}^{2}=2 / 3$. Thus, $\ddot{B}$ should turn to negative at some earlier time and subsequently the Hubble radius increases (see figure).

Here, one should also check that in this period $\dot{B}^{2}$ given in (35) is always positive. To see this is indeed the case, let us define $f=\sigma e^{-2 B}$ and $g=V /\left(6 \sqrt{1-\dot{T}^{2}}\right)$. Then, initially $f(0)=g(0)$. From (37) we find that

$$
\frac{d \ln g}{d t}=\frac{d \ln f}{d t}\left(\frac{3 \dot{T}^{2}}{2}\right) \text {. }
$$

Since initially $\dot{B}(0)=0$ and $\ddot{B}(0)>0$, there is an interval during which $\dot{B}>0$ and consequently $d(\ln f) / d t<0$. Also from (37), we have $d(\ln g) / d t<0$. Then (38) implies $d(\ln g) / d t>d(\ln f) / d t$ since initially $\dot{T} \ll 1$. Therefore, $g>f$ during this period and from (35) one sees $\dot{B}^{2}>0$. Iterating, it is guaranteed that $\dot{B}^{2}>0$ until $\dot{T}^{2}=2 / 3$.

As a result, we see that it is possible to obtain a cosmological phase with decreasing Hubble radius which can be matched to the static strongly coupled Hagedorn phase, provided that the flat space is replaced by a sphere, the tachyon field dominates the cosmic evolution and the initial tachyon potential energy is fine tuned with the curvature of the three sphere. Of course, it should be checked that these assumptions do not invalidate the results of [26-29]. Especially, since the curvature of the sphere in- 
troduces a new length scale, it may alter the scale independence of the perturbation spectrum. Moreover, the existence of the tachyon field should be explained. In any case, we find it interesting that a slight modification of the tachyon cosmology yields a decreasing Hubble radius.

\section{Acknowledgments}

The work of Ali Kaya is partially supported by Turkish Academy of Sciences via Young Investigator Award Program (TÜBA-GEBIP).
[1] R. Branderberger and C. Vafa, "Superstrings in the early universe", Nucl. Phys. B316, 391, (1989).

[2] T. Battefeld and S. Watson, "String Gas Cosmology", Rev. Mod. Phys. 78 (2006) 435, hep-th/0510022.

[3] R. H. Brandenberger, "Moduli Stabilization in String Gas Cosmology", Prog. Theor. Phys. Suppl. 163 (2006) 358, hep-th/0509159.

[4] M. Majumdar and A.-C. Davis, "D-brane Anti-brane Annihilation in an Expanding Universe", JHEP 0312 (2003) 012, hep-th/0304153.

[5] R. Easther, B.R. Greene, M.G. Jackson and D. Kabat, "String windings in the early universe", JCAP 0502 (2005) 009, hep-th/0409121.

[6] R. Danos, A.R. Frey, A. Mazumdar, "Interaction Rates in String Gas Cosmology", Phys. Rev. D70 (2004) 106010, hep-th/0409162.

[7] A. Campos, "Late-time dynamics of brane gas cosmology", Phys. Rev. D68 (2003) 104017, hep-th/0304216.

[8] S. Watson and R. Brandenberger, "Stabilization of Extra Dimensions at Tree Level", JCAP 0311 (2003) 008, hepth/0307044.

[9] S.P. Patil and R. Brandenberger, "Radion Stabilization by Stringy Effects in General Relativity and Dilaton Gravity", Phys. Rev. D71 (2005) 103522, hepth/0401037.

[10] S.P. Patil and R.H. Brandenberger, "The Cosmology of Massless String Modes", JCAP 0601 (2006) 005, hepth/0502069.

[11] S.P. Patil, "Moduli (Dilaton, Volume and Shape) Stabilization via Massless F and D String Modes", hepth/0504145.

[12] R. Brandenberger, Y.-K. E. Cheung and S. Watson, "Moduli Stabilization with Long Winding Strings", JHEP 0605 (2006) 025, hep-th/0501032.

[13] S. Alexander , R.H. Brandenberger and D. Easson, "Brane gases in the early universe", Phys. Rev. D62, 103509, (2000), hep-th/0005212.

[14] D. A. Easson, "Brane Gases on K3 and Calabi-Yau Manifolds", Int. J. Mod. Phys. A18 (2003) 4295, hepth/0110225.

[15] S.H.S. Alexander, "Brane Gas Cosmology, M-theory and Little String Theory", JHEP 0310 (2003) 013, hepth/0212151.

[16] A. Kaya, "Volume Stabilization and Acceleration in Brane Gas Cosmology", JCAP 08 (2004) 004, hepth/0405099.

[17] S. Arapoglu, A. Kaya, "D-brane Gases and Stabilization of Extra Dimensions in Dilaton Gravity", Phys. Lett. B603 (2004) 107, hep-th/0409094.

[18] T. Rador, "Intersection Democracy for Winding Branes and Stabilization of Extra Dimensions", Phys. Lett. B621 (2005) 176, hep-th/0501249.

[19] T. Rador, "Vibrating Winding Branes, Wrapping Democracy and Stabilization of Extra Dimensions in Dilaton Gravity", JHEP 0506 (2005) 001, hepth/0502039.

[20] T. Rador, "Stabilization of extra dimensions and the dimensionality of the observed space", hep-th/0504047.

[21] A. Kaya, "Brane gases and stabilization of shape moduli with momentum and winding stress", Phys. Rev. D72, 066006, 2005, hep-th/0504208.

[22] A. Berndsen and J. Cline, "Dilaton Stabilization in brane gas cosmology", hep-th/0408185, Int. J. Mod. Phys.A19, 5311, 2004, hep-th/0408185.

[23] A. Berndsen, T. Biswas and J.M. Cline "Moduli Stabilization in Brane Gas Cosmology with Superpotentials", JCAP 0508 (2005) 012, hep-th/0505151.

[24] D. A. Easson and M. Trodden, "Moduli Stabilization and Inflation Using Wrapped Branes", Phys. Rev. D72 (2005) 026002, hep-th/0505098.

[25] S. Cremonini and S. Watson, "Dilaton Dynamics from Production of Tensionless Membranes", Phys. Rev. D73 (2006) 086007, hep-th/0601082.

[26] A. Nayeri, R.H. Brandenberger and C. Vafa, "Producing a Scale-Invariant Spectrum of Perturbations in a Hagedorn Phase of String Cosmology", Phys. Rev. Lett. 97 (2006) 021302, hep-th/0511140.

[27] A. Nayeri, "Inflation Free, Stringy Generation of ScaleInvariant Cosmological Fluctuations in $\mathrm{D}=3+1$ Dimensions", hep-th/0607073.

[28] R.H. Brandenberger, A. Nayeri, S.P. Patil and C. Vafa, "String Gas Cosmology and Structure Formation", hepth/0608121.

[29] R.H. Brandenberger, S. Kanno, J. Soda, D.A. Easson, J. Khoury, P. Martineau, A. Nayeri and S. Patil, "More on the Spectrum of Perturbations in String Gas Cosmology", hep-th/0608186.

[30] N. Kaloper, L. Kofman, A. Linde and V. Mukhanov, "On the new string theory inspired mechanism of generation of cosmological perturbations", hep-th/0608200 .

[31] A. A. Tseytlin and C. Vafa, "Elements of String Cosmology", Nucl. Phys. B372 (1992) 443, hep-th/9109048.

[32] J.H. Schwarz, "An SL(2,Z) multiplet of type IIB superstrings", hep-th/9508143, Phys. Lett. 360B, 13,1995, hep-th/9508143.

[33] E. Witten, "Bound states of strings and p-branes", Nucl.Phys. 460B, 335, 1996, hep-th/9510135.

[34] G.W. Gibbons, "Cosmological evolution of the rolling tachyon", Phys. Lett. 537B, 1, 2002, hep-th/0204008. 